\title{
A PROOF OF THE IMPOSSIBILITY OF COMPLETING INFINITELY MANY TASKS
}

BY

JEREMY GWIAZDA

\begin{abstract}
In this article, I argue that it is impossible to complete infinitely many tasks in a finite time. A key premise in my argument is that the only way to get to 0 tasks remaining is from 1 task remaining, when tasks are done 1-by-1. I suggest that the only way to deny this premise is by begging the question, that is, by assuming that supertasks are possible. I go on to present one reason why this conclusion (that supertasks are impossible) is important, namely that it implies a new verdict on a decision puzzle propounded by Jeffrey Barrett and Frank Arntzenius.
\end{abstract}

We are not so naïve as to think that we have had the last word on supertasks. Since it is the business of philosophers to uncover logical and conceptual difficulties, we would not be surprised if there were to be continued assertions that supertasks are by their very nature contradictory, paradoxical, or puzzling - not surprised, but certainly disappointed (Earman and Norton, 1996, p. 257).

Is it logically possible to complete infinitely many tasks in a finite time? Though a classic example of such a supertask is Thomson's Lamp (Thomson, 1970), let us imagine a simpler case. There are tennis balls numbered with each positive integer. Ball 1 is burned at time $=1 / 2$, ball 2 at $\mathrm{t}=3 / 4$, ball 3 at $\mathrm{t}=7 / 8, \ldots$ Is it logically possible to burn all of the tennis balls? The standard response is to argue that it is, as for any ball, it was burned at some time prior to $t=1$, and so no balls remain at $t=1$. Thus infinitely many balls were burned. In what follows I attempt to give a proof that it is impossible to complete infinitely many tasks 1-by-1.

Pacific Philosophical Quarterly 93 (2012) 1-7

(C) 2012 The Author

Pacific Philosophical Quarterly (C) 2012 University of Southern California and Blackwell Publishing Ltd. 
1. By definition, completing infinitely many tasks requires getting the number of tasks remaining down to 0 .

2. If tasks are done 1-by-1, then the only way to get to 0 tasks is from 1 task, because if more than 1 task remains, then performing a task does not leave 0 tasks. (This reasoning holds in both the finite and infinite cases.)

3. When infinitely many tasks are attempted 1-by-1, there is no point at which 1 task remains.

4. Then from 2 and 3, there is no point at which 0 tasks remain.

5. Then from 1 and 4 , it is not possible to complete infinitely many tasks.

This proof trades on the intuition that in order to complete some number of tasks 1-by-1, it is necessary to 'go through' every smaller number of tasks, and in particular, the only way to get to 0 tasks is from 1 task. When it is claimed that infinitely many tasks are completed, this principle fails, in that at all times $<1$ infinitely many tasks remain, and then at time $=1,0$ tasks remain (it is claimed). We never 'went through' the finite numbers, and in particular, 1. It is not enough for the defender of the possibility of completing infinitely many tasks to simply retell the story, 'perform task 1 at $\mathrm{t}=1 / 2$, task 2 at $\mathrm{t}=3 / 4, \ldots$.' Rather, the defender must find the flaw in the five-step argument above. To successfully defend the logical possibility of completing infinitely many tasks, some direct response to this challenge is needed. ${ }^{1}$

A first objection to my argument is that I am mistakenly assuming that $\omega$ has a last member. But I am doing no such thing. I am giving an argument that a task ${ }^{2}$ of order-type $\omega$, a supertask, cannot be completed. And of course, a reason for this can be that $\omega$ has no last member. Certainly it is reasonable that the order-type of the task matters as to whether or not it is completable. But, to reiterate, I am not assuming that $\omega$ has a last member, which it does not. ${ }^{3}$

A second objection is that it is obviously logically possible to complete infinitely many tasks in a finite time. And indeed, retelling the story of the supertask does suggest that supertasks are logically possible. That is, I am able to put on my defend-the-logical-possibility-of-completing-infinitelymany-tasks hat and reason along the following lines:

Premise 2 is simply false in the infinite case. As is well known, the infinite differs from the finite. It is not necessary to 'go through' 1 task when attempting to complete infinitely many. Here is how it is possible to complete infinitely many tasks: do task 1 at $\mathrm{t}=1 / 2$, task 2 at $\mathrm{t}=3 / 4, \ldots$ Then infinitely many tasks are completed in a finite time.

And I do, in fact, feel pulled by such reasoning. But I also feel compelled by the five-step argument. Certainly the infinite differs from the finite, but 
why should premise 2 fail in the infinite case (especially when performing 1 task when infinitely many remain leaves infinitely many tasks)? Far from failing in the infinite case, premise 2 is true in both the finite and infinite cases. That is, I suggest that when we ask: How is it possible to get to 0 tasks when performing tasks 1-by-1?, the only sensible answer is: From 1 task. Assuming that supertasks are possible does contradict premise 2, but by begging the question (the entire issue we are investigating is whether or not supertasks are logically possible). I offer the following picture in support of premise 2 :

$$
\infty \rightarrow \infty \quad \ldots .3 \rightarrow 2 \rightarrow 1 \rightarrow 0
$$

What the picture indicates is that when a task is performed when there are infinitely many tasks, then infinitely many tasks remain (' $\infty \rightarrow \infty$ '). The right side of the picture shows that when a task is performed when, e.g., there are 2 tasks, then 1 task remains. How do we get to 0 tasks? From 1 task, and only from 1 task. There is no other way; premise 2 reflects this reasoning.

Note that based on the picture above, specifically $\infty \rightarrow \infty$, it is unclear how infinitely many tasks could ever be completed. Let us now turn to a story that highlights this concern. Imagine that hardworking Hans and lazy Lori undertake the tennis ball supertask described above. Hans performs the supertask exactly as described. Lori however, being lazy, does the following. She observes Hans. Whenever he acts (burns a tennis ball) she asks: Based on Hans's latest action, is my task still order-isomorphic ${ }^{4}$ to Hans's remaining task? If not, she burns a tennis ball to restore isomorphism; she does so prior to Hans's next burning. If so, being a good Cantorian and lazy, she does nothing, content that her task is still the same as Hans's. At time = 1, how have things gone wrong for Lori? That is, she consciously kept her task order-isomorphic to Hans's task. Lori never burned a tennis ball precisely because Hans never seemed to make any progress. Whenever Hans burned a tennis ball, he went from a task of order-type $\omega$ to a task of order-type $\omega$. And Lori's task, of order-type $\omega$, was in every case order-isomorphic. How then, are the tasks different at time $=1$ ? That is, on the standard view (that supertasks are possible) Hans has no balls remaining; Lori has infinitely many. But I have suggested that Lori's task should be order-isomorphic to Hans's, and so, in particular, the two should have the same number of balls remaining. Where did lazy Lori go wrong?

Let us consider two potential responses. The first is that there is not spatio-temporal continuity through this completed supertask. But note that we allowed spatio-temporal continuity in the case where Hans alone was burning tennis balls. It is then unclear why the introduction of lazy Lori, who does nothing, would lead to a breakdown of spatio-temporal continuity. A second response is to suggest that there is no problem. Hans 
completes his supertask; Lori does not. Let me then reiterate what I take to be the problem.

Again note that a task of order-type $\omega$ is order-isomorphic to a task of order-type $\omega$. Then, every action Hans performs takes him from a task of order-type $\omega$ to a task of order-type $\omega$; he never seems to make any progress (recall: $\infty \rightarrow \infty$ ). And so Lori has a good question when she asks, 'How did Hans complete his supertask? Hans never made any progress with any particular burning. How then can infinitely many cases of no progress lead to the completion of a supertask? Adding nothing (or, no progress) infinitely many times is still nothing.' Hans can reply, 'Well, burning ball 1 is progress; there is one less ball to be burned.' But we should note that this is a Euclidean view (the part is smaller than the whole) and not a Cantorian view (order-isomorphic tasks are equivalent). Perhaps it is dangerous to be both lazy and Cantorian. And I suggest that even on a Euclidean view, it remains mysterious as to exactly how lazy Lori went awry in her effort to keep her task order-isomorphic to hardworking Hans's, as every time Hans burned a ball, Lori subsequently verified that her task was still equivalent.

To this point I have argued that it is not possible to complete a supertask. What is the importance of this conclusion? In the remainder of the article, I outline one answer to this question. In 'An Infinite Decision Puzzle,' Jeffrey Barrett and Frank Arntzenius (1999, p. 101) present a case where, 'one is guaranteed to do better than a seemingly rational agent by always acting in what appears to be an entirely irrational way.' Their example (1999, pp. 101-102) is as follows:

Suppose one has an infinite stack of dollar bills with consecutive serial numbers: 1, 2, 3, etc. An agent, who starts with no money, is then offered the following choice, where $n$ is equal to the total number of times that the choice has been offered so far: 1) Get one dollar off the top of the stack. [Or,] 2) Get $2^{\text {n+1 }}$ dollar bills off the top of the stack, but you must return the bill with the smallest serial number that you currently have . . Now suppose the agent is offered the choice at $1 / 2$ minute, at $3 / 4$ minute, at 7/8 minute, at 15/16 minute, etc.

Barrett and Arntzenius claim that with choice 1) the agent ends up with an infinite amount of money, whereas with choice 2), the agent winds up with no money. The commonly accepted reasoning in case 2) is that, despite the fact that the agent's money is growing rapidly prior to one minute, at one minute every dollar bill was returned at some time, and thus the agent is left with 0 dollars; however, intuitions on this puzzle diverge. The reasoning just outlined is accepted by, e.g., Ross (1988), Allis and Koetsier (1991), Earman and Norton (1996, pp. 239-241), and Barrett and Arntzenius (1999). By contrast, Clark (2007, pp. 147-148) writes, ' . . . the state at the limit, [one minute], is not determined' and van Bendegem (1994, p. 743) writes, '. . . it seems to me that RP [Ross' Paradox] must be considered an impossible supertask much like the Thomson Lamp supertask.' 
To my knowledge, no one has argued that an agent does better opting for choice 2). But if supertasks cannot be completed, then choice 2) is the rational choice. Note that the completion of an infinite task in a finite time (a supertask) is required in order to generate Barrett and Arntzenius' result that 1) is preferable to 2). Above I suggested that it is not possible to complete supertasks. Though currently an unpopular position, many others have argued (or presumably would have argued) that it is not possible to complete supertasks. Aristotle, Wittgenstein, and Brouwer come to mind as notable historical examples. For example, Mark van Atten (2004, p. 6) discusses Brouwer's ontology, writing, 'The very insight that this process of embedding two-ities can be iterated gives rise to the infinite ordinal $\omega$ (the first number after $1,2,3 \ldots$. . This infinity has to be thought of as potential and not actual: on the one hand, the creating subject can start a potentially infinite task, but on the other hand, it cannot complete it.' More generally, those who reject the actual infinite and those who link mathematics with human mental activity will tend to find the completion of supertasks impossible. In what follows, I present a way to model supertasks (such as the supertasks described by Barrett and Arntzenius) assuming that supertasks cannot be completed. On this modeling, choice 2) will emerge as the rational choice as compared with 1).

If supertasks cannot be completed, as I have argued above, then there is no state after infinitely many tasks. Finitely many tasks can be completed, and this number grows without bound. If this is the case, how can we model Barrett and Arntzenius' examples 1) and 2)? I suggest the following. At each stage, an agent has some amount of money. Let the agent have $n_{i}$ dollars at stage $\mathrm{i}$. Then, the agent can be said to tend to $\left[<\mathrm{n}_{1}, \mathrm{n}_{2}, \mathrm{n}_{3}, \ldots>\right]$ dollars, where $\left[<\mathrm{n}_{1}, \mathrm{n}_{2}, \mathrm{n}_{3}, \ldots>\right.$ ] is a hyperreal, as discussed in Goldblatt, 1991. Thus in 1), the agent tends to $[<1,2,3, \ldots>]$ dollars. In example 2 ), the agent tends to $[<3,10,25, \ldots>]$ dollars. Then, as $[<3,10,25, \ldots>]$ is greater than $[<1,2,3, \ldots>]$, option 2$)$ is the rational choice. Why does this modeling make sense if supertasks cannot be completed? We are assuming that the agent will not stop at any finite point, and so it does not make sense to say that the agent has a finite amount of money in 1) or 2). ${ }^{5}$ However, as infinitely many tasks are never actually completed, it also does not make sense to discuss the state after infinitely many tasks. Thus it makes sense to model the situation using hyperreals, and to say that the agent's money tends to an infinite value, where the infinite values can differ [as 1) and 2) differ]. Such modeling works in other cases. For example, if agent3 takes 2 dollars per stage, then agent3's money tends to $[<2,4$, $6, \ldots>$ ] dollars, which is better than option 1), but worse than 2). Let agent4's money tend to $[<3,3,7,7,11,11 \ldots>$ dollars. Then it is not clear whether agent 3 or agent 4 is preferable, and happily the ultrafilter will 'decide' which infinite hyperreal is larger. ${ }^{6}$ I suggest that hyperreals can be used as outlined, and that 2) is the rational choice over 1) (again, if 
supertasks cannot be completed). And if 2) is preferable to 1), then Barrett and Arntzenius' conclusions regarding rationality do not succeed. That is, the agent who makes rational choices at each stage [option 2)] outperforms the agent who opts for less money at each stage [option 1)]. Thus the issue of the completability of supertasks not only determines which mathematical model is the correct one to use to model this situation, but also has implications regarding rationality.

Many people argue that there is no problem with the completion of infinitely many tasks in a finite time, and even express disappointment in those who suggest otherwise. ${ }^{7}$ In this article, I have argued that the completion of a supertask is impossible because at no point does 1 task remain, and the only way to arrive at 0 tasks remaining is via 1 task. It seems obvious that performing a task when more than 1 task remains does not get one to 0 tasks. Assuming that supertasks are completable simply begs the question against this argument. Thus I suggest that supertasks are more problematic than many people believe, and also that this incompletability of supertasks is important. ${ }^{8}$

\section{Department of Philosophy \\ Lehman College}

\section{NOTES}

${ }^{1}$ As I came to the completion of this article, I found an article with a similar thesis to mine. To a certain extent the argument presented in Groarke (1982) anticipated my argument, and I believe that his article deserves more attention than it has received (to my knowledge it has only been cited once). On page 73 of his article, Groarke discusses, 'PSA (The Principle of Sequential Acts): The performance of a sequence of acts does not complete a particular task unless it is completed by the performance of one of the acts in the sequence.' Note also that these arguments are not confined to the completion of infinitely many tasks in a finite time, but also rule out the possibility of completing infinitely many tasks in an infinite time. In this article, however, I focus on supertasks.

${ }^{2}$ Note that I sometimes use 'task' to refer to a single burning of a tennis ball, and sometimes, as in this sentence, I use 'task' to refer to the entire sequence of tasks. Context makes clear which meaning is intended.

${ }^{3}$ It does follow from my argument that a necessary, but not sufficient, condition for a sequence of tasks to be completable is that the sequence has a last task. And so $\omega$ tasks (supertasks) are not completable. But neither are a $\omega+1$ tasks.

${ }^{4}$ Tasks are order-isomorphic when there is an order preserving bijection between the two tasks. So a task of order-type $\omega$ is order-isomorphic to any other task of order-type $\omega$, but not to, e.g., tasks of order-type $0,7, \omega+1$, etc.

${ }^{5}$ Though of course, at any one specific time the agent does have a finite amount of money.

${ }^{6}$ That is, I am suggesting that the arbitrary choice of an ultrafilter is actually a good feature of this modeling.

${ }^{7}$ See the quote at the beginning of the article.

${ }^{8}$ I thank an anonymous referee for comments and suggestions that benefitted the article. 


\section{REFERENCES}

Allis, V. and Koetsier, T. (1991). 'On Some Paradoxes of the Infinite,' British Journal for the Philosophy of Science 42, pp. 187-194.

van Atten, M. (2004). On Brouwer (Wadsworth Philosophers Series). Belmont, CA: Wadsworth/Thomson Learning.

Barrett, J. and Arntzenius, F. (1999). 'An Infinite Decision Puzzle,' Theory and Decision 46, pp. 101-103.

van Bendegem, J. P. (1994). 'Ross' Paradox is an Impossible Supertask,' British Journal for the Philosophy of Science 45, pp. 743-748.

Clark, M. (2007). Paradoxes from A to $Z, 2^{\text {nd }}$ edn. New York: Routledge.

Earman, J. and Norton, J. (1996). 'Infinite Pains: The Trouble with Supertasks,' in A. Morton and S. Stich (eds), Benacerraf and His Critics. Cambridge: Blackwell.

Goldblatt, R. (1991). Lectures on the Hyperreals. New York: Springer.

Groarke, L. (1982). 'Zeno's Dichotomy: Undermining the Modern Response,' Auslegung 9, pp. 67-75.

Ross, S. (1988). A First Course in Probability, $3^{\text {rd }}$ edn. New York: Macmillan.

Thomson, J. (1970). 'Tasks and Super-Tasks,' in W. Salmon (ed) Zeno's Paradoxes. Indianapolis, IN: Hackett. 